\title{
KLF2 and KLF4 control endothelial identity and vascular integrity
}

\author{
Panjamaporn Sangwung, ${ }^{1,2}$ Guangjin Zhou, ${ }^{1}$ Lalitha Nayak, ${ }^{1,3}$ E. Ricky Chan, ${ }^{4}$ Sandeep Kumar, ${ }^{5}$ \\ Dong-Won Kang, ${ }^{5}$ Rongli Zhang, ${ }^{1}$ Xudong Liao, ${ }^{1}$ Yuan Lu, ${ }^{1}$ Keiki Sugi, ${ }^{1}$ Hisashi Fujioka, ${ }^{6}$ Hong Shi, \\ Stephanie D. Lapping, ${ }^{1}$ Chandra C. Ghosh, ${ }^{7}$ Sarah J. Higgins, ${ }^{7}$ Samir M. Parikh, ${ }^{7}$ Hanjoong Jo, ${ }^{5,8}$ \\ and Mukesh K. Jain ${ }^{1,2,9}$
}

'Cardiovascular Research Institute, Department of Medicine, and 'Department of Physiology and Biophysics, Case Western Reserve University School of Medicine, Cleveland, Ohio, USA. ${ }^{3}$ Division of Hematology and Oncology, University Hospitals Cleveland Medical Center, Case Western Reserve University, Cleveland, Ohio, USA. ${ }^{4}$ nstitute for Computational Biology, Case Western Reserve University School of Medicine, Cleveland, Ohio, USA. ${ }^{5}$ Wallace H. Coulter Department of Biomedical Engineering, Georgia Institute of Technology and Emory University, Atlanta, Georgia, USA. ${ }^{6}$ Electron Microscopy Core Facility, Case Western Reserve University School of Medicine, Cleveland, Ohio, USA. 'Center for Vascular Biology Research and Department of Medicine, Beth Israel Deaconess Medical Center and Harvard Medical School, Boston, Massachusetts, USA. ${ }^{8}$ Division of Cardiology, Emory University, Atlanta, Georgia, USA. ${ }^{9}$ Harrington Heart and Vascular Institute, University Hospitals Cleveland Medical Center, Cleveland, Ohio, USA.

Maintenance of vascular integrity in the adult animal is needed for survival, and it is critically dependent on the endothelial lining, which controls barrier function, blood fluidity, and flow dynamics. However, nodal regulators that coordinate endothelial identity and function in the adult animal remain poorly characterized. Here, we show that endothelial KLF2 and KLF4 control a large segment of the endothelial transcriptome, thereby affecting virtually all key endothelial functions. Inducible endothelial-specific deletion of KIf2 and/or KIf4 reveals that a single allele of either gene is sufficient for survival, but absence of both (EC-DKO) results in acute death from myocardial infarction, heart failure, and stroke. EC-DKO animals exhibit profound compromise in vascular integrity and profound dysregulation of the coagulation system. Collectively, these studies establish an absolute requirement for KLF2/4 for maintenance of endothelial and vascular integrity in the adult animal.

Authorship note: P. Sangwung and G. Zhou contributed equally to this work.

Conflict of interest: The authors have declared that no conflict of interest exists.

Submitted: November 10, 2016 Accepted: January 5, 2017 Published: February 23, 2017

\section{Reference information:} JCI Insight. 2017;2(4):e91700. https:// doi.org/10.1172/ji.insight.91700

\section{Introduction}

The maintenance of an intact vascular network to deliver oxygen and nutrients to all tissues is critical for organismal survival. The endothelium is critical to vascular integrity by virtue of its ability to control fundamental properties such as permeability, blood fluidity, and vasomotor tone (1). The endothelium is also a dynamic and highly responsive tissue whose function can be altered by biomechanical (e.g., blood flow) and biochemical (e.g., cytokine) stimuli (2). For example, laminar blood flow alters cellular gene expression in a manner that promotes a healthy endothelium and maintains vascular integrity while disturbed flow confers antiparallel effects $(3,4)$. Further, biochemical stimuli, such as cytokines, can activate the endothelium, as seen in acute (e.g., sepsis) or chronic disease states (e.g., coronary artery disease), culminating in leakage of fluid from the intravascular space and tissue edema, formation of blood clots that impair flow, and altered vascular tone resulting in blood pressure dysregulation (2). Given the importance of vascular integrity in organismal survival, the identification of nodal regulators is of interest.

Studies over the past decade have led to the appreciation that members of the Kruppel-like family of transcription factors (KLFs) regulate endothelial biology (5). In particular, two members of this family - namely KLF2 and KLF4 - are enriched in the endothelium, regulated by flow and cytokines, and have been shown in cell-based studies to directly regulate key endothelial genes, such as endothelial nitric oxide synthase (Nos3) and thrombomodulin (Thbd) (6-9). In vivo studies using systemic deletion of KLF2 and/or KLF4 result in death during embryonic development or shortly after birth (10-13). Hemizygous or conditional global deletion of $\mathrm{KLF} 2$ alters vascular blood-brain barrier functions $(14,15)$. 
Finally, endothelial deficiency of KLF4 was found to render animals susceptible to atherosclerosis and thrombosis and injury-induced neointimal formation, underscoring the importance of this gene family in vessel biology $(16,17)$. However, the importance of having two KLFs with seemingly overlapping functions in the adult animal has not been illuminated and remains a major question in the field. Here, using inducible gene deletion approaches we have deleted endothelial Klf2, Klf4, or both in the adult organism. Our results demonstrate an absolute requirement for both factors in control of cardinal endothelial functions and maintenance of an intact vasculature.

\section{Results}

Endothelial-specific Klf2 and Klf4 deletion leads to rapid death of adult mice. To evaluate the importance of endothelial KLF2 and KLF4 in adult vasculature, we generated mouse models that allow for inducible endothelial-specific deletion of Klf2 (EC-Klf2-KO), Klf4 (EC-Klf4-KO), Klf2 plus one allele of Klf4 (EC-Klf2-KO/Klf4+/-), Klf4 plus one allele of Klf2 (EC-Klf4-KO/Klf2+/-), or both genes (EC-DKO) through the tamoxifen-mediated activation of Cdh5(PAC)-Ert2cre (CRE). Deletion of Klf2 and Klf4 as well as reduced expression of well-established downstream targets, such as Nos3 and Thbd, was confirmed in primary microvascular endothelial cells (EC) isolated from the heart at day 4 to 6 after tamoxifen injection (Supplemental Figure 1, A-D; supplemental material available online with this article; doi:10.1172/jci.insight.91700DS1). Strikingly, following tamoxifen injection, EC-DKO mice succumbed starting from day 6 after injection (40\% of mortality rate) and reached a 100\% mortality rate at day 9 (Figure 1A). By contrast, expression of one allele of Klf2 or Klf4 was sufficient for survival. Thus, we focused our analysis on EC-DKO mice.

To glean insights into the cause of acute death, we performed continuous telemetry monitoring before and after tamoxifen injection. Electrocardiogram (EKG) recording revealed that EC-DKO animals experienced a progressive reduction in heart rate and elevation of $\mathrm{T}$ wave area (an indicator of ischemic heart injury) (Figure 1, B and C, and Supplemental Figure 2). Echocardiographic analysis in EC-DKO mice at day 6 revealed a significant decrease in cardiac function, as evidenced by reduced left ventricular fractional shortening, ejection fraction, and cardiac output (Figure 1D and Supplemental Videos 1 and 2). In addition, significant left ventricular hypertrophy and dilation (Supplemental Figure 3, B and C), increased circulating cardiac troponin I (indicative of myocyte injury; Figure 1E), and cardiomyocyte death (Figure $1 \mathrm{~F}$ ) were observed in EC-DKO mice. By contrast, the EKG and cardiac function were not significantly altered in EC-Klf2-KO and EC-Klf4-KO mice (Supplemental Figures 2 and 3). Finally, gross postmortem examination revealed small-vessel hemorrhages in the brain, lungs, heart, and subcutaneous tissues of EC-DKO animals but not those of CRE or single-knockout animals (Figure $1 G$ and Supplemental Figure 4). Consonant with these observations, EC-DKO mice exhibited stroke-like symptoms (e.g., partial loss of voluntary movement and weakness in the limbs; Supplemental Videos 3 and 4). These results indicate that endothelial deletion of Klf2 and Klf4 led to compromised vasculature, which manifested as cardiac failure, neurologic dysfunction, and death.

Endothelial-specific deletion of Klf2 and Klf4 leads to vascular leak and systemic coagulopathy. To understand the basis for widespread small-vessel hemorrhage in multiple organs, we hypothesized that alterations in fundamental properties, such as barrier function and/or blood coagulation, may be operative. Histological analyses of brain and lung tissues revealed extravasation of blood into adjacent tissues (Figure 2A, top row, and Supplemental Figure 5A). Electron micrographs demonstrated loss of EC contiguity (Figure 2A, middle row; Supplemental Figure 5A, and Supplemental Figure 7). To gain a quantitative assessment of vascular permeability, we injected control and EC-DKO mice with Evans Blue dye (EBD) and examined the tissues for extravasation at day 4-6 after tamoxifen injection. Significant EBD accumulation in the extravascular tissues of the lungs and kidneys was obvious in EC-DKO mice at day 4-5 (Supplemental Figure 5, B and $\mathrm{C}$ ) and the brain, lungs, kidneys, and heart at day 6 (Figure 2A, bottom row; and Supplemental Figure 5, A and D). Consistent with these observations, qPCR analyses revealed a marked reduction in a number of tight junction and adherens junction genes in ECs from EC-DKO mice (Figure 2B). Finally, previous studies have shown that increased angiopoietin-2 (Angpt-2) contributes to vascular leak (18-20). As shown in Figure 2C, circulating Angpt-2 levels were markedly increased in EC-DKO mice.

Another etiology for widespread, multiorgan hemorrhage is alteration in the hemostatic and coagulation systems. To assess the coagulation system, we first performed a complete blood count after tamoxifen injection. While no effect was seen on white blood cell or red blood cell counts, a significant reduction in platelets (thrombocytopenia) was observed in EC-DKO animals (Figure 2D). Further, microscopic examination of the periph- 
A
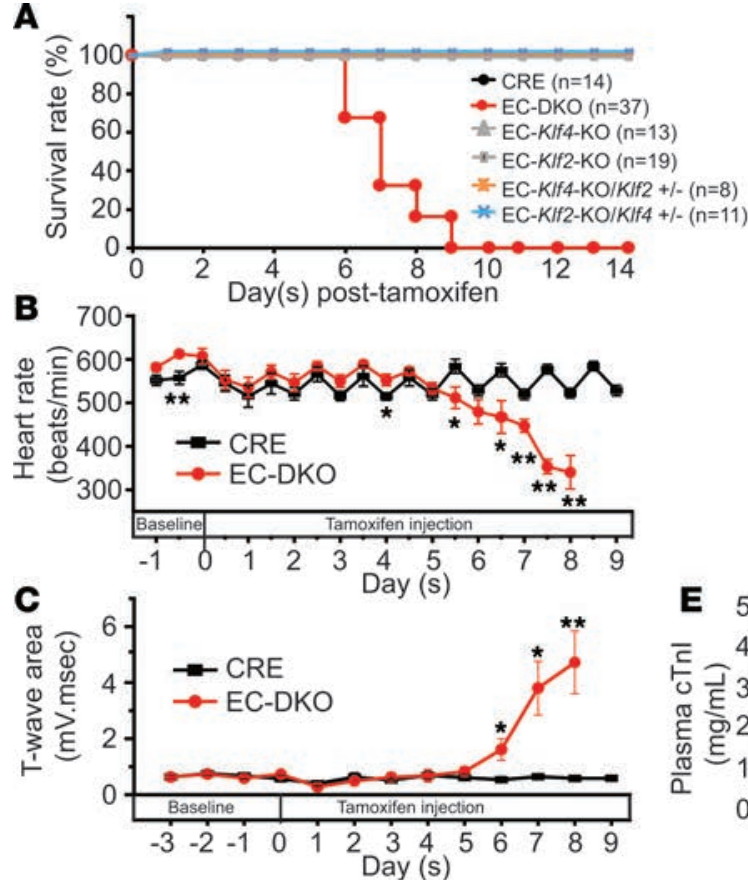

$\mathbf{F}$

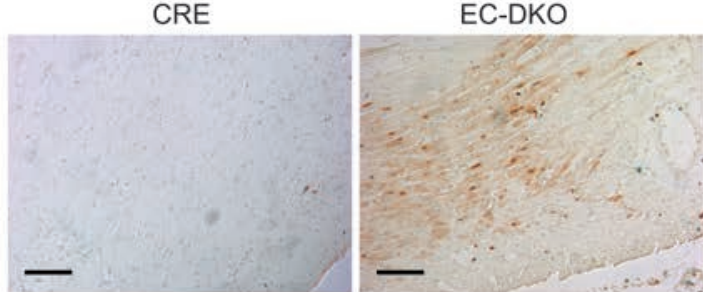

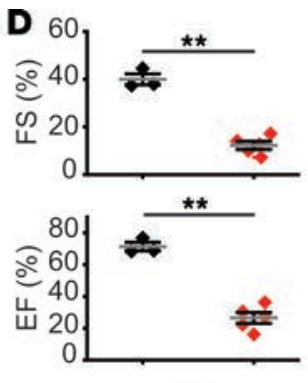

$\mathbf{G}$
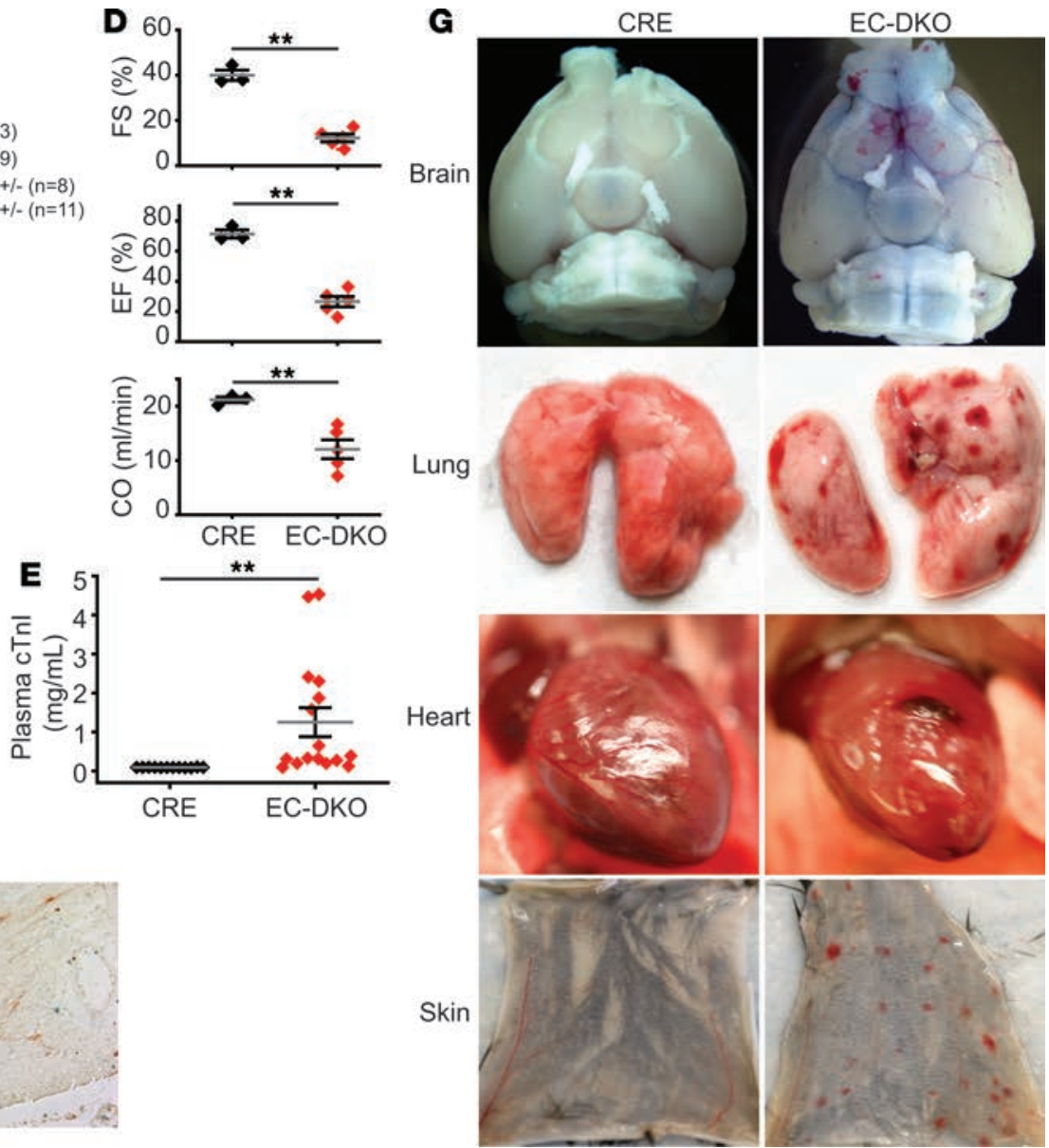

Figure 1. Endothelial-specific KIf2 and KIf4 deletion leads to rapid death of adult mice. (A) Survival curve of tamoxifen-induced endothelial-specific KIf2 and/or KIf4 gene deletion in adult mice. $n=37$ EC-specific KIf2 and KIf4 double knockout (EC-DKO); $n=14$ Cdh5(PAC)-Ert2cre (CRE); $n=19$ EC-specific KIf2 knockout (EC-KIf2-KO); $n=13$ EC-specific KIf4 knockout (EC-KIf4-KO), $n=8$ EC-specific knockout of KIf4 plus one allele of KIf2 (EC-KIf4-KO/KIf2+/-); $n=11$, EC-specific knockout of KIf2 plus one allele of KIf4 mice (EC-KIf2-KO/KIf4+/-). (B) Reduction of heart rate and (C) ST wave segment elevation were observed in EC-DKO mice ( $n=4-5$ per genotype). (D) Fractional shortening (FS), ejection fraction (EF), and cardiac output (CO) in CRE ( $n=3$ ) and EC-DKO mice $(n=5)$ at day 6 after tamoxifen. (E) Circulating cardiac troponin I level $(n=11-16$ per genotype) and (F) representative images of TUNEL staining in the heart showing massive cardiomyocyte death in EC-DKO mice at day 6 after tamoxifen ( $n=3$ per genotype). Scale bar: $100 \mu \mathrm{m}$. (C) Representative gross anatomy images of brain, lungs, heart, and subcutaneous tissues at day 6 after tamoxifen indicate spontaneous hemorrhage in EC-DKO mice ( $n=3-4$ per genotype). EC, endothelial cell. Data are presented as mean $\pm \mathrm{SEM}$ values. ${ }^{*} P<0.05,{ }^{* *} P<0.01$. 2 -tailed Student's $t$ test.

eral blood smear revealed the presence of fragmented red blood cells (i.e., schistocytes) in EC-DKO animals at day 6 after tamoxifen injection (Figure 2E). Given the combination of low platelet counts and fragmented red blood cells, we posited that the precipitous drop in platelets was likely secondary to excessive consumption, a scenario seen in coagulopathic states such as disseminated intravascular coagulation (21). Indeed, blood coagulation studies revealed significant elevations in prothrombin, activated partial thromboplastin times, and plasma D-dimer levels in EC-DKO mice (Figure 2, F and G), indicating dysregulation of coagulation.

Intact blood flow depends upon a fine balance between circulating and endothelial procoagulant and anticoagulant factors. Our group previously reported that both KLF2 and KLF4 induced the expression of thrombomodulin $(8,9)$, the key blood-clotting regulator in the protein $C$ anticoagulation pathway that is expressed in the endothelium $(22,23)$. We observed a near-complete loss of thrombomodulin expression both in mRNA and protein levels (Supplemental Figure 1, A-D) in the ECs isolated from EC-DKO mice at day 6 after tamoxifen. We also looked at other factors in procoagulation and anticoagulation pathways. While expression of tissue factor (F3) was not significantly changed, levels of the serine (or cysteine) peptidase inhibitor, clade E, member 1 (Serpine1) and coagulation factor II receptor-like 3 (F2rl3) were increased in the EC-DKO mice (Supplemental Figure 6). The above results provide evidence for the presence of a coagulopathic condition on day 6 after tamoxifen injection in EC-DKO mice that is likely secondary to the decline in known antithrombotic factors 
A

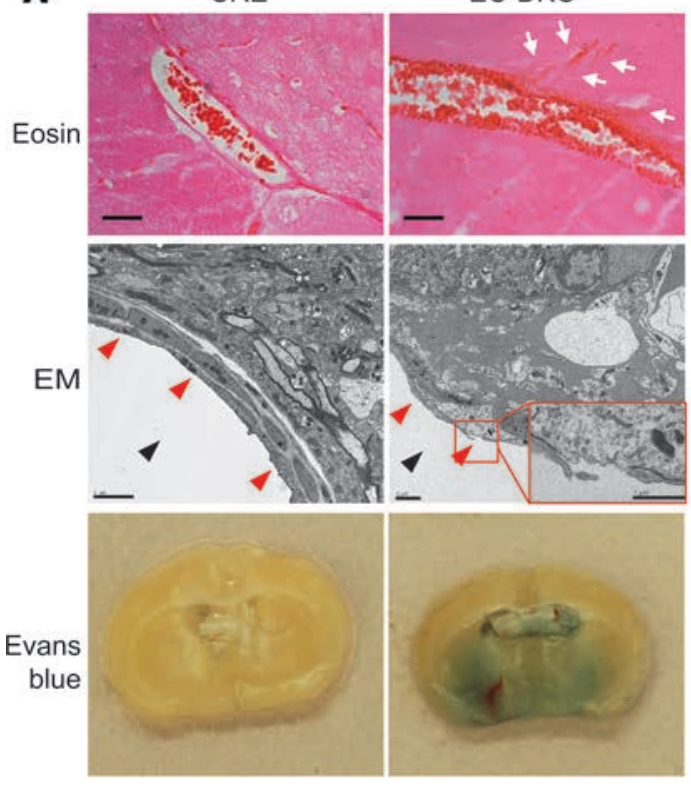

B

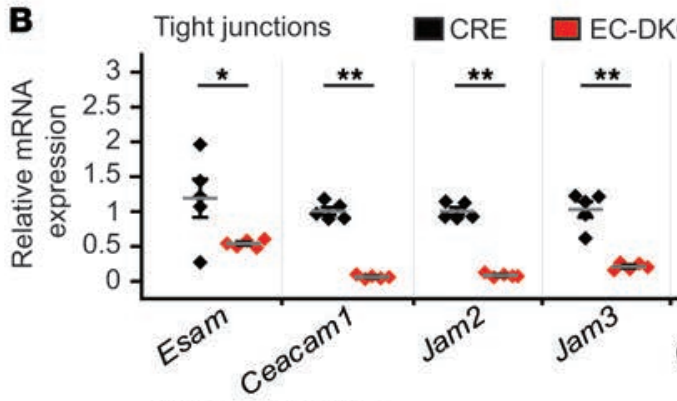

Adherens junctions

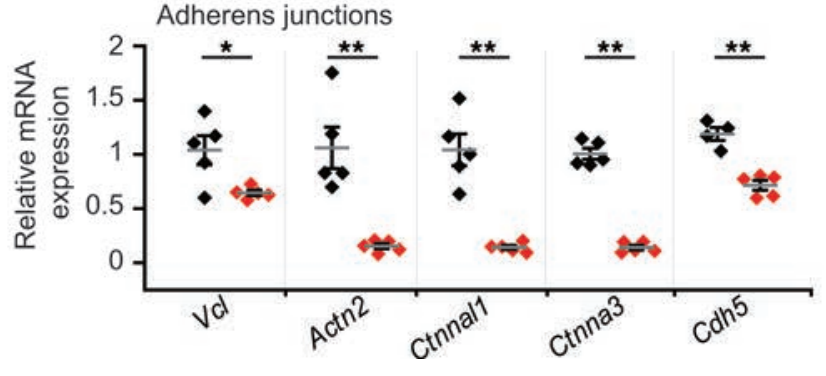

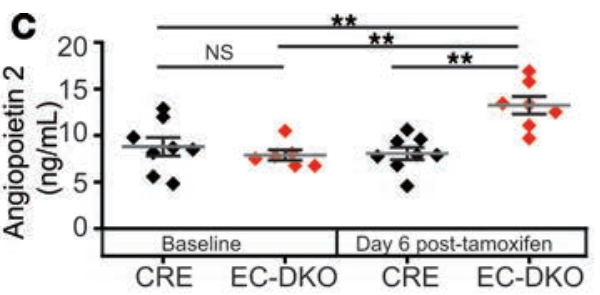

E

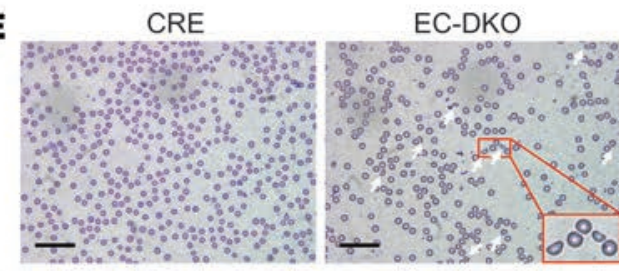

F Extrinsic pathway, PT Intrinsic pathway, aPTT

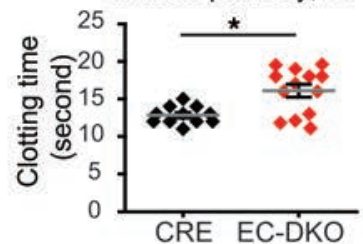

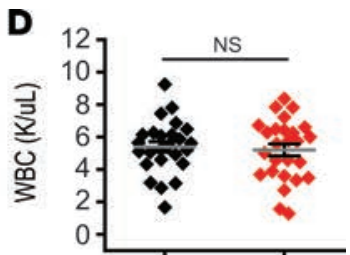
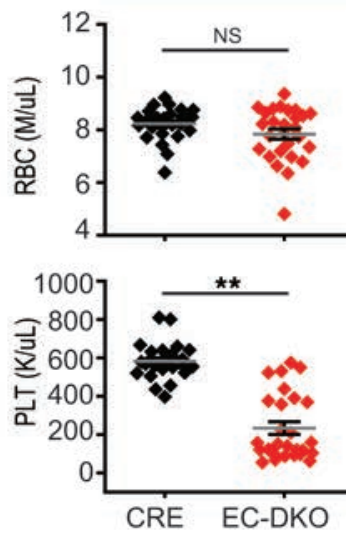

Figure 2. Endothelial-specific deletion of $\boldsymbol{K I f 2}$ and $\boldsymbol{K} \mathbf{I f} \mathbf{4}$ leads to vascular leak and systemic coagulopathy. (A, top) Representative images of brain eosin staining show erythrocyte leakage (white arrows) in extravascular tissues at day 6 after tamoxifen ( $n=3$ per genotype). Scale bar: $50 \mu \mathrm{m}$. (A middle) Representative electron microscopic (EM) images of brain indicate discontinuity of endothelial monolayer in the EC-DKO mice at day 6 after tamoxifen ( $n=3-4$ per genotype). Scale bar: $2 \mu \mathrm{m}$. Endothelial cells (EC), red arrowheads; vessel lumen, black arrowheads. The box outlined in red is shown at high magnification in the inset. Scale bar: $1 \mu \mathrm{m}$. (A, bottom) Representative images of Evans blue vascular permeability assay of the brain at day 6 after tamoxifen ( $n=7-8$ per genotype). (B) Relative mRNA expression levels of endothelial tight and adherens junction genes in primary cardiac microvascular ECs 6 days after tamoxifen injection ( $n=3-5$ per genotype, each sample was pooled from 2 mice). (C) Circulating angiopoietin-2 protein levels before and after tamoxifen ( $n=6-8$ per genotype). (D) White blood cell (WBC), red blood cell (RBC), and blood platelet (PLT) counts at day 6 after tamoxifen ( $n=25-29$ per genotype). (E) Representative images of blood smear in CRE and EC-DKO mice ( $n=3$ per genotype). White arrows indicate erythrocyte fragmentation. Scale bar: $50 \mu \mathrm{m}$. The box outlined in red is shown at high magnification in the inset. (F) Extrinsic and intrinsic pathway coagulation assays ( $n=11-13$ per genotype). PT, prothrombin; aPTT, activated partial thromboplastin times. (G) Plasma D-dimer levels at day 6 after tamoxifen (CRE, $n=5$; EC-DKO, $n=6$ ). CRE, Cdh5(PAC)-Ert2cre; EC-DKO, EC-specific KIf2 and KIf4 double knockout. Data are presented as mean \pm SEM. ${ }^{*} P<0.05 ;{ }^{*} P<0.01$. Two-way ANOVA with Tukey's post-hoc test and 2-tailed Student's $t$ test. 
A 6,094 significantly differentially expressed genes

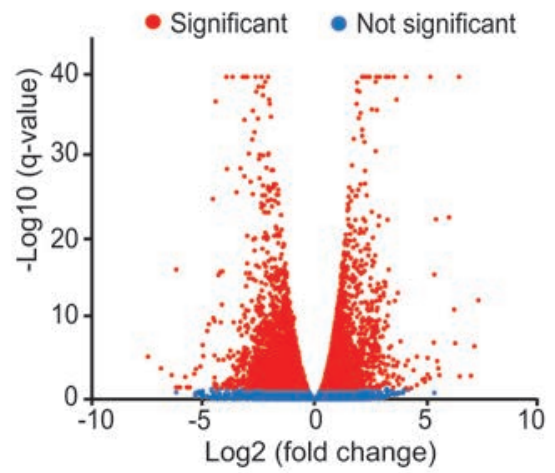

B

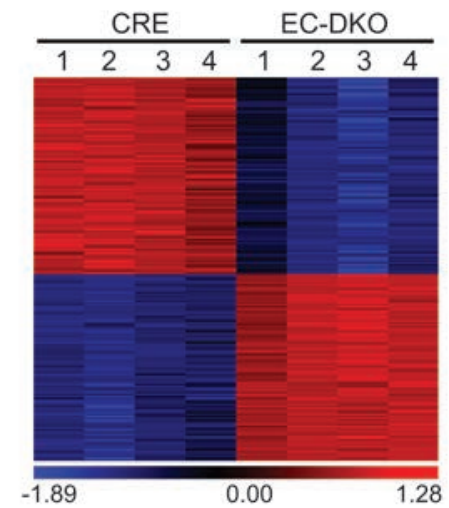

C Hallmark Pathways Normalized Enrichment Score (NES)

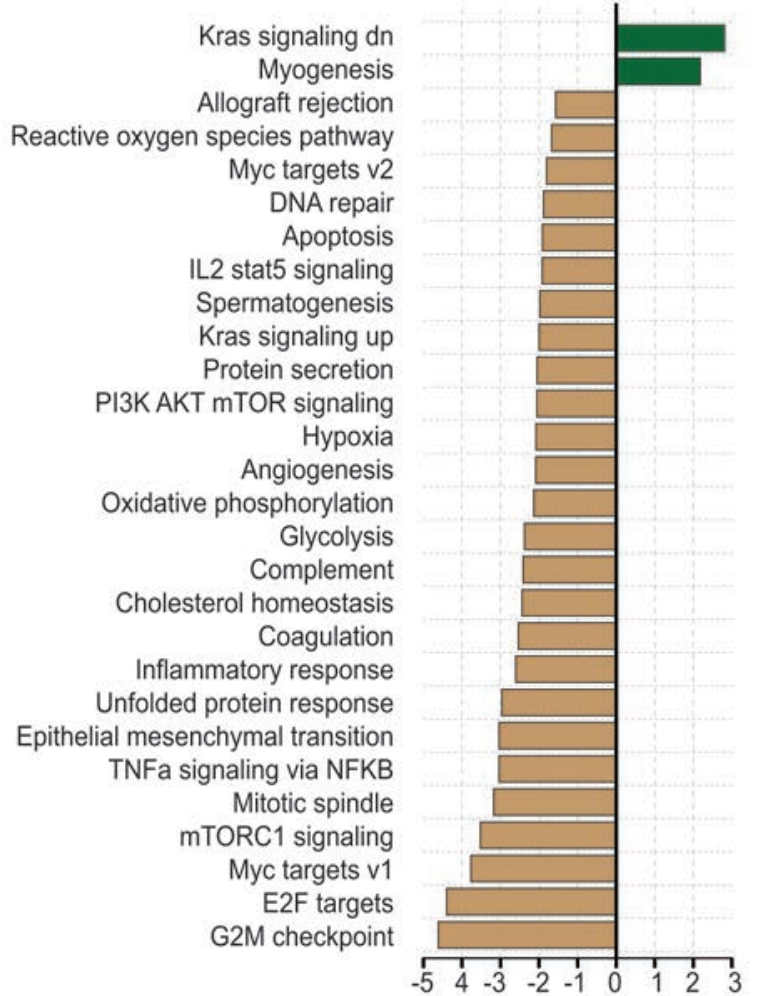

Figure 3. Endothelial-specific KIf2 and KIf4 deletion results in profound alterations in the EC transcriptome. (A) A volcano plot showing 6,094 significantly differentially expressed genes (red dots indicate $q<0.05$ ) in primary cardiac microvascular endothelial cells (EC) obtained from EC-DKO mice when compared with CRE mice. Significantly differentially expressed genes were determined by Cuffdiff. (B) Heatmap of the top 200 genes differentially expressed in primary cardiac microvascular ECs of CRE and EC-DKO mice ( $n=4$ per genotype, each $n$ was pooled from 2 mice). (C) Normalized enrichment score (NES) represents the distribution of 28 of the 50 hallmark gene pathways. Positive and negative values indicate upregulation of genes in the CRE mice in comparison to EC-DKO mice and upregulation of genes in EC-DKO mice relative to CRE mice, respectively. CRE, Cdh5(PAC)-Ert2cre; EC-DKO, EC-specific KIf2 and KIf4 double knockout.

such as thrombomodulin. This acquired deficiency in thrombomodulin is similar to observations in genetic models of thrombomodulin deficiency $(23,24)$ and suggests that loss of this critical factor on ECs likely contributes to development of a prothrombotic milieu, resulting in widespread consumption of coagulation factors and platelets, leading to generalized bleeding.

Endothelial-specific Klf2 and Klf4 deletion results in profound alterations in the EC transcriptome. Given that dual loss of KLF2 and KLF4 led to a profound compromise of the most fundamental properties of the vasculature, we posited that the alteration in gene expression may be quite significant. Unbiased transcriptomic analyses in mouse cardiac microvascular ECs derived from CRE and EC-DKO mice at the beginning of day 6 after tamoxifen injection demonstrated extensive differences in the endothelial transcriptome (6,094 genes with $q<$ 0.05 ; Figure 3, A and B, and Supplemental Table 1). By contrast, we identified a small number of differentially expressed genes in EC-Klf2-KO (45 genes with $q<0.05$; Supplemental Table 2) and EC-Klf4-KO (30 genes with $q<0.05$; Supplemental Table 3) mice when compared with CRE animals. Gene set enrichment analysis of ECs from DKO mice revealed marked alterations $(q<0.05)$ in 28 of the 50 hallmark gene pathways (Figure 3C), while no significant changes were noted in single-knockout mice. These observations indicate that loss of both KLF2 and KLF4 profoundly altered the endothelial transcriptomic landscape.

\section{Discussion}

Evolutionary studies suggest that the vascular system arose approximately 600 million years ago to overcome the time-distance constraints of diffusion critical advance for metazoan life (25). The endothelium arose to provide barrier function, localize immune/coagulation functions, and optimize flow to help 
overcome diffusion constraints to ensure adequate supply of oxygen and nutrients to all tissues. In this study, we advance that view that two transcription factors, KLF2 and KLF4, govern these ancient and fundamental properties of the endothelium and vascular integrity.

We demonstrated, for the first time to our knowledge, that loss of both endothelial KLF2 and KLF4 expression in adult animals resulted in acute vascular dysfunction and death. The profound vascular defects seen in EC-DKO mice are likely a combined result of vascular barrier disruption as well as a generalized state of consumptive coagulopathy. Although widespread damage to the endothelium or vascular barrier (secondary to junction proteins) can trigger consumptive coagulation, our results demonstrating early, substantial loss of thrombomodulin suggest that acquired thrombomodulin deficiency is an important contributing factor to the development of a consumptive coagulopathy. This is consistent with previous murine studies wherein endothelial-specific thrombomodulin deletion resulted in cerebellar thrombohemorrhagic strokes, generalized thrombosis, and disseminated intravascular coagulation $(23,24)$. Further, RNA sequencing data showed changes of genes $(q<0.05)$ in the coagulation pathway (Supplemental Table 4$)$, and examination of other factors important for hemostasis revealed significant elevation in Serpine1 and F2rl3 expression, predisposing to the development of a prothrombotic milieu. Thus, both EC-autonomous function and altered vascular barrier function likely contribute to the full phenotype observed in EC-DKO mice. We also note that our findings are consistent with but add significantly to previous findings. Specifically, Chiplunkar et al. (13) reported the role of both factors in regulating vascular integrity using systemic knockout embryos of both Klf2 and Klf4 genes. However, their study used a global deletion of Klf2 and Klf4 genes in the embryos and focused on early development. In contrast, our study specifically abrogated both Klf2 and Klf4 genes in ECs in adult animals. Thus, the present study definitively highlights the absolute requirement of these two KLF factors to maintain normal EC function and consequently vessel integrity in the adult animal.

Another important aspect of our work relates to the observation that a single allele of either Klf2 or Klf4 is sufficient for maintenance of intact vasculature and survival. These data provide insights into a long-standing question in the field regarding redundancy between KLF2 and KLF4. Indeed, we and others have reported that both factors are induced by the same stimuli (e.g., flow and statins) and that both regulate similar key endothelial targets (e.g., Nos3 and Thbd) to confer antiinflammatory and antithrombotic effects to the vessel wall $(4,6,9,16,26)$. Given the obvious requirement for an intact vasculature, it is likely that the redundancy of $\mathrm{KLF} 2 / 4$ provides an important safety net to ensure survival. However, the fact that one allele is sufficient for survival does not necessarily mean that the vasculature is healthy. Indeed, endothelial loss of KLF4 alone renders animals susceptible to chronic inflammation and atherothrombosis and injury-induced neointimal formation $(16,17)$. Further, previous studies showed that loss of either KLF2 or KLF4 renders the vasculature susceptible to leakage in response to inflammatory or ischemic injury $(14,27)$. Thus, it is likely that mice lacking 3 of 4 alleles will be susceptible to vascular dysfunction under stress conditions.

Another key finding relates to the breadth of the endothelial transcriptome under control of KLF2/4. It is hypothesized that, while the genome consists of approximately 20,000 genes (28), most cells express approximately $50 \%-60 \%$ of these at any given time. The large transcriptomic alterations seen in ECs deficient in KLF2 and KLF4 suggest that these 2 factors control a large segment of the active endothelial transcriptome, affecting numerous cellular processes, indicating a profound landscape-level change in EC identity and function. A broad change in gene expression was observed at the beginning of day 6 after tamoxifen, reflective of changes before catastrophic functional collapse. That said, we cannot rule out the possibility that the interaction of ECs with other cells and plasma components may contribute. Future studies assessing RNA sequencing at day 3-5 may be helpful in this regard. Collectively, our findings establish endothelial KLF2 and KLF4 as master regulators of endothelial biology and vascular integrity.

\section{Methods}

Animal models. All animals are of the C57BL6/J mouse strain. Endothelial-specific Klf2 and/or Klf4 knockout mice were generated by breeding floxed mice $(K l f 2 f / f$, $K l f 4 f / f$, or $K l f 2 f / f-K l f 4 f / f)$ with CRE mice (originally from R. Adams, University of Münster, Münster, Germany). Endothelial-specific deletion of Klf2 plus one allele of Klf4 (EC-Klf2-KO/Klf4+/-) mice were generated by mating Cdh5(PAC)Ert2cre-Klf2 $f / f-K l f 4 f / f$ mice with Cdh5(PAC)-Ert2cre-Klf2 $f / f$ mice. Endothelial-specific deletion of Klf4 plus one allele of Klf2 (EC-Klf4-KO/Klf2+/-) mice were generated by mating Cdh5(PAC)-Ert2cre$K l f 2 f / f-K l f 4 f / f$ mice with Cdh5(PAC)-Ert2cre-Klf4 $f / f$ mice. To trigger endothelial-specific deletion of Klf2 and/or Klf4 gene deletion, 8- to 10-week-old mice were intraperitoneally injected with tamoxifen 
(2 mg/25 g) (MP Biomedicals). Cdh5(PAC)-Ert2cre mice were used as a control group. The survival study was conducted in both male and female mice. Other studies were performed in male mice.

$E C$ isolation. Primary microvascular ECs were isolated from the heart tissue of mice using a standard technique as previously described with a minor modification $(16,29)$. Briefly, the hearts were washed in cold PBS, minced with blades, and digested in PBS containing 1\%BSA, collagenase type I, $1 \mathrm{mM} \mathrm{CaCl}{ }_{2}$, and $1 \mathrm{mM} \mathrm{MgCl}_{2}$ at $37^{\circ} \mathrm{C}$ for 45 minutes. ECs were purified by using Dynabeads (Thermo Fisher Scientific) conjugated with anti-CD31 antibody (catalog 553370, BD Biosciences). The cells were immediately subjected to further experiments without culturing them on a petri dish. We isolated ECs by mechanical mincing and enzymatic digestion to obtain single-cell suspension of the tissues. ECs were separated from the rest of the cells and were isolated by CD31-coated magnetic beads. Although CD31 is expressed on platelets and subsets of leukocytes in addition to ECs, its expression on ECs is higher than on platelets and subsets of leukocytes (30). In addition, CD31 is one of the two most specific antibodies used for isolation of ECs, and purity of the EC fraction sorted by CD31 antibody is $>95 \%$ (31).

$R N A$ isolation and $q P C R$. Total RNA from ECs was isolated by using the High Pure RNA Isolation Kit and reverse transcribed into cDNA using the iScript cDNA Synthesis Kit (Bio-Rad). Quantitative real-time PCR (qPCR) was carried out using TaqMan Master Mix (Applied Biosystems), and the reactions were performed using the StepOnePlus Real-Time PCR System (Applied Biosystems). Endogenous GAPDH was used as a normalizer for gene expression. The primer sequences used are listed in Supplemental Table 5. Relative mRNA expression levels (fold changes) between groups were calculated using the $\delta$ - $\delta$ Ct method.

$R N A$ sequencing and bioinformatics analysis. The quality of RNA isolated from cardiac microvascular ECs ( $n=4$ per group, each $n$ was pooled from 2 mice) was measured by Agilent 2200 TapeStation, and all samples have an RNA integrity number $>7.4$. Total RNA samples were submitted to the UCSF genomics core laboratories for RNA sequencing. Library preparation was employed using the Illumina TruSeq Stranded Total RNA kit. Sequencing was performed by using the Illumina SE 50-bp platform. Reads were aligned to the mouse genome (Ensembl Mouse GRCm38) using tophat. Reads that mapped to known mRNAs were subjected to differential expression analysis. A total of 6,094 genes were determined to be differentially expressed between the DKO and control group using a $q$ value of less than 0.05 (FDR-corrected $P$ value). This list was filtered for genes with a $>2$-fold change, which narrowed our list to 2,743 genes. This list was used as the input for our gene set enrichment analysis using the GenePattern toolset (Broad Institute). Pathways with a $P$ value correction with FDR $<0.05$ were considered significant. RNA sequencing data have been deposited in the NCBI's Gene Expression Omnibus and are accessible through GEO series accession number GSE92965 (https://www.ncbi.nlm.nih. gov/geo/query/acc.cgi?acc=GSE92965).

Echocardiogram. At days 6 after tamoxifen administration, animals under anesthesia were placed on a temperature-controlled platform with heart rate and respiratory rate monitoring. Ultrasound gel was applied on the furless chest, and noninvasive images were captured when the heart rate was stable (500 beats/minute) by using a 14-MHz ultrasound probe that was connected to a VisualSonic Vevo 770 machine.

Telemetry measurement and ST wave analysis. Animals were anesthetized by isoflurane. A sterilized DSI wireless telemetric transmitter (DSI) was implanted into the abdominal cavity. Animals were monitored on a telemetry receiver, and a baseline measurement (before tamoxifen injection) was conducted. Two days following the implantation, animals were injected with tamoxifen, and data were perpetually recorded for 9 days. ST wave analysis was conducted using LabChart7 software.

Evans blue vascular permeability assay. After tamoxifen injection for 4 to 6 days, animals were anesthetized with ketamine/xylazine ( $170 \mathrm{mg} / \mathrm{kg}$ and $5 \mathrm{mg} / \mathrm{kg}$, respectively) via intraperitoneal injection. One hundred $\mu 1$ of PBS containing 1\% EBD (Sigma-Aldrich) was administered via retro-orbital injection, and EBD was allowed to circulate in the body for 2 hours. Excess EBD in the vasculature and blood was removed via transcardial perfusion of PBS containing $2 \mathrm{mM}$ EDTA for 8 minutes. The peristaltic pump was set at $4 \mathrm{ml} / \mathrm{minute}$. The excised tissues were homogenized in formamide (Sigma-Aldrich), and EBD was extracted from the homogenized tissues by incubating at $70^{\circ} \mathrm{C}$ for 24 hours. Following a centrifugation at $12,000 \mathrm{~g}$ for 30 minutes, the absorbance of EBD in the supernatant was measured at $620 \mathrm{~nm}$ (the absorbance maximum for EBD) and corrected for a contamination of heme pigments (OD740 nm) as follows: OD620 (corrected) $=$ OD620 $-(1.326 \times$ OD740) +0.03 .

Peripheral blood count, blood smear, coagulation, and fibrinogen assays. Blood was collected from the inferior vena cava using 3.2\% sodium citrate as an anticoagulant, and peripheral blood count was carried out by using an automated analyzer (Hemavet 950FS). A blood smear was stained with Wright's stain, and 
the images were captured by a Leica DM2000 LED microscope (Leica Microsystems Inc.). Whole blood was centrifuged at 2,000 $\mathrm{g}$ for 15 minutes at room temperature, and plasma was collected. Coagulation assays for the extrinsic and intrinsic pathways (prothrombin time and activated partial thromboplastin time, respectively) were carried out as described in the manufacturer's instructions (Helena Laboratories). Plasma fibrinogen was measured by using the fibrinogen assay kit (Helena Laboratories).

ELISA. Plasma cardiac troponin I (Life Diagnostics Inc.), D-Dimer (Cloud-Clone Corp), and Angpt-2 (Abcam) levels were determined as described in the manufacturers' instructions.

Western blot analysis. Primary cardiac microvascular ECs were homogenized and lysed for 10 minutes at $4^{\circ} \mathrm{C}$ in RIPA buffer (Thermo Fisher Scientific) containing proteinase inhibitor cocktail. The lysed cells were centrifuged at $15,871 \mathrm{~g}$ for 10 minutes at $4^{\circ} \mathrm{C}$, and the supernatant was collected. Protein was separated on $4 \%-20 \%$ Tris-Glycine gel and transferred onto Nitrocellulose membrane. The membrane was blotted with primary antibodies against KLF4 (catalog AF3640, R\&D Systems Inc.), eNOS (catalog 610296, BD Biosciences), TM (catalog sc-7097, Santa Cruz Biotechnology Inc.), and GAPDH (catalog G9545, Sigma-Aldrich). Secondary antibodies include horseradish peroxidase-conjugated anti-rabbit IgG (catalog 7074), anti-mouse IgG (catalog 7076, Cell Signaling Technology Inc.), and anti-goat IgG (catalog sc-2020, Santa Cruz Biotechnology Inc.)

Immunohistochemistry. Formalin-fixed, paraffin-embedded tissues were cut in 8 - $\mu$ m-thick sections and mounted on coverslips for eosin staining. Apoptosis staining of ventricles (5- $\mu \mathrm{m}$-thick sections) was assessed using the ApopTag Peroxidase In Situ Apoptosis Detection Kit (Millipore) as described in manufacturer's instructions. Tissue images were taken on a Leica DM2000 LED microscope.

Transmission electron microscopy. Animals were anesthetized and 1\% lidocaine was injected through abdominal aorta toward the left to open the valves and relax the smooth muscle cells. Animals were perfused transcardially with triple aldehyde-DMSO at a flow rate of $10 \mathrm{ml} /$ minute for 10 minutes. Brain and lungs were isolated from CRE and EC-DKO mice at day 6 after tamoxifen injection. Small pieces of the brain and lung tissue were fixed for 2 hours at room temperature by immersion in the freshly prepared triple aldehyde-DMSO (32). After rinsing in 0.1 M HEPES buffer ( $\mathrm{pH} 7.3$ ), they were postfixed in ferrocyanide-reduced osmium tetroxide. Another water rinse was followed by an overnight soak in acidified uranyl acetate. After again rinsing in distilled water, the tissue blocks were dehydrated in ascending concentrations of ethanol, passed through propylene oxide, and embedded in Poly/Bed resin (Polysciences). Thin sections were sequentially stained with acidified uranyl acetate followed by a modification of Sato's triple lead stain (33). These sections were examined in a FEI Tecnai Spirit (T12) transmission electron microscope with a Gatan US4000 4k $\times 4 \mathrm{k}$ CCD.

Statistics. Data are presented as mean \pm SEM. Statistical analyses were performed using 2-tailed Student's $t$ test and ANOVA with post-hoc test for multiple comparisons to analyze the difference between 2 groups and among the groups, respectively. A $P$ value equal to or less than 0.05 was considered significant.

Study approval. All animal studies were approved by the Case Western Reserve University IACUC.

\section{Author contributions}

MKJ, LN, HJ, and PS designed the studies. PS, GZ, LN, SK, DWK, YL, HF, HS, SDL, CCG, and SJH performed the experiments. RZ performed animal surgeries. KS performed echocardiogram. PS, GZ, RZ, ERC, LN, XL, YL, KS, HF, SK, CCG, and SJH analyzed the data. MKJ, XL, YL, SMP, and HJ provided research guidance. PS prepared the figures and drafted the manuscript. MKJ edited the manuscript and approved final version of the manuscript.

\section{Acknowledgments}

This work was supported by NIH grants R01HL110630-01, R01HL112486, R01HL086548, and R01HL119195 (to MKJ); R01HL093234 and R01HL125275 (to SMP); and K08HL121131-01 (to LN) as well as by American Heart Association grants 12SDG12050558 (to YL), 12SDG12070077 (to XL), and 16POST31200017 (to SJH).

Address correspondence to: Mukesh K. Jain, Cardiovascular Research Institute, Case Western Reserve University School of Medicine, 2103 Cornell Road, Cleveland, Ohio 44106, USA. Phone: 216.368.2036; E-mail: mxj84@case.edu. 
1. Aird WC. Phenotypic heterogeneity of the endothelium: I. Structure, function, mechanisms. Circ Res. 2007;100(2):158-173.

2. Aird WC. Endothelium as an organ system. Crit Care Med. 2004;32(5 suppl):S271-S279.

3. Heo KS, Fujiwara K, Abe J. Disturbed-flow-mediated vascular reactive oxygen species induce endothelial dysfunction. Circ $J$. 2011;75(12):2722-2730.

4. Jain MK, Sangwung P, Hamik A. Regulation of an inflammatory disease: Krüppel-like factors and atherosclerosis. Arterioscler Thromb Vasc Biol. 2014;34(3):499-508.

5. Atkins GB, Jain MK. Role of Kruppel-like transcription factors in endothelial biology. Circ Res. 2007;100(12):1686-1695.

6. Dekker RJ, et al. Prolonged fluid shear stress induces a distinct set of endothelial cell genes, most specifically lung Kruppel-like factor (KLF2). Blood. 2002;100(5):1689-1698.

7. Parmar KM, et al. Integration of flow-dependent endothelial phenotypes by Kruppel-like factor 2. J Clin Invest. 2006;116(1):49-58.

8. Hamik A, et al. Kruppel-like factor 4 regulates endothelial inflammation. J Biol Chem. 2007;282(18):13769-13779.

9. Lin Z, et al. Kruppel-like factor 2 (KLF2) regulates endothelial thrombotic function. Circ Res. 2005;96(5):e48-e57.

10. Kuo CT, Veselits ML, Barton KP, Lu MM, Clendenin C, Leiden JM. The LKLF transcription factor is required for normal tunica media formation blood vessel stabilization during murine embryogenesis. Genes Dev. 1997;11(22):2996-3006.

11. Segre JA, Bauer C, Fuchs E. Klf4 is a transcription factor required for establishing the barrier function of the skin. Nat Genet. 1999;22(4):356-360.

12. Basu P, et al. KLF2 is essential for primitive erythropoiesis regulates the human murine embryonic beta-like globin genes in vivo. Blood. 2005;106(7):2566-2571.

13. Chiplunkar AR, et al. The Krüppel-like factor 2 and Krüppel-like factor 4 genes interact to maintain endothelial integrity in mouse embryonic vasculogenesis. BMC Dev Biol. 2013;13:40.

14. Lin Z, et al. Kruppel-like factor 2 regulates endothelial barrier function. Arterioscler Thromb Vasc Biol. 2010;30(10):1952-1959.

15. Shi H, et al. Kruppel-like factor 2 protects against ischemic stroke by regulating endothelial blood brain barrier function. Am $J$ Physiol Heart Circ Physiol. 2013;304(6):H796-H805.

16. Zhou G, et al. Endothelial Kruppel-like factor 4 protects against atherothrombosis in mice. J Clin Invest. 2012;122(12):4727-4731.

17. Yoshida T, Yamashita M, Horimai C, Hayashi M. Deletion of Krüppel-like factor 4 in endothelial and hematopoietic cells enhances neointimal formation following vascular injury. J Am Heart Assoc. 2014;3(1):e00622.

18. Roviezzo F, et al. Angiopoietin-2 causes inflammation in vivo by promoting vascular leakage. J Pharmacol Exp Ther. 2005;314(2):738-744

19. Benest AV, et al. Angiopoietin-2 is critical for cytokine-induced vascular leakage. PLoS One. 2013;8(8):e70459.

20. Gallagher DC, et al. Angiopoietin 2 is a potential mediator of high-dose interleukin 2-induced vascular leak. Clin Cancer Res. 2007;13(7):2115-2120.

21. Levi M, Toh CH, Thachil J, Watson HG. Guidelines for the diagnosis and management of disseminated intravascular coagulation. British Committee for Standards in Haematology. Br J Haematol. 2009;145(1):24-33.

22. Healy AM1, Rayburn HB, Rosenberg RD, Weiler H. Absence of the blood-clotting regulator thrombomodulin causes embryonic lethality in mice before development of a functional cardiovascular system. Proc Natl Acad Sci U S A. 1995;92(3):850-854.

23. Weiler-Guettler H, Aird WC, Husain M, Rayburn H, Rosenberg RD. Targeting of transgene expression to the vascular endothelium of mice by homologous recombination at the thrombomodulin locus. Circ Res. 1996;78(2):180-187.

24. Isermann B, et al. Endothelium-specific loss of murine thrombomodulin disrupts the protein C anticoagulant pathway and causes juvenile-onset thrombosis. J Clin Invest. 2001;108(4):537-546.

25. Monahan-Earley R1, Dvorak AM, Aird WC. Evolutionary origins of the blood vascular system and endothelium. J Thromb Haemost. 2013;11(suppl 1):46-66.

26. SenBanerjee S, et al. KLF2 Is a novel transcriptional regulator of endothelial proinflammatory activation. J Exp Med. 2004;199(10):1305-1315.

27. Cowan CE, Kohler EE, Dugan TA, Mirza MK, Malik AB, Wary KK. Kruppel-like factor-4 transcriptionally regulates VE-cadherin expression and endothelial barrier function. Circ Res. 2010;107(8):959-966.

28. International Human Genome Sequencing Consortium. Finishing the euchromatic sequence of the human genome. Nature. 2004;431(7011):931-945.

29. Lim YC, et al. Heterogeneity of endothelial cells from different organ sites in T-cell subset recruitment. Am J Pathol. 2003;162(5):1591-1601.

30. Wang Y, Sheibani N. Expression pattern of alternatively spliced PECAM-1 isoforms in hematopoietic cells and platelets. $J$ Cell Biochem. 2002;87(4):424-438.

31. van Beijnum JR, Rousch M, Castermans K, van der Linden E, Griffioen AW. Isolation of endothelial cells from fresh tissues. Nat Protoc. 2008;3(6):1085-1091.

32. Fujioka H, Tandler B, Hoppel CL. Mitochondrial division in rat cardiomyocytes: an electron microscope study. Anat Rec (Hoboken). 2012;295(9):1455-1461.

33. Hanaichi T, Sato T, Iwamoto T, Malavasi-Yamashiro J, Hoshino M, Mizuno N. A stable lead by modification of Sato's method. J Electron Microsc (Tokyo). 1986;35(3):304-306. 